\title{
Effects of the Rates on the Performance and Pressure Pulsations of a High-Speed Pump with Straight Blade
}

\author{
Hao Jia, Xianghui Su $\mathbb{D}^{\text {, }}$, and Wei Jin \\ Key Laboratory of Fluid Transmission Technology of Zhejiang Province, Zhejiang Sci-Tech University, Hangzhou 310018, China \\ Correspondence should be addressed to Xianghui Su; suxianghui@zstu.edu.cn
}

Received 17 September 2021; Accepted 20 November 2021; Published 8 December 2021

Academic Editor: Diansen Yang

Copyright (c) 2021 Hao Jia et al. This is an open access article distributed under the Creative Commons Attribution License, which permits unrestricted use, distribution, and reproduction in any medium, provided the original work is properly cited.

\begin{abstract}
Along with the crucial requirement for efficiency improvement in the cutting-edge petrochemical technology, the evaluation of the dynamic performance characteristics of high-speed pump is becoming increasingly important. It has become a main topic in the research of high-speed pump to minimize the pressure pulsation induced by the fluid in the pump body, so as to reduce the mechanical vibration. Although the research on the transient flow characteristic and pressure fluctuation of a high-speed pump with straight blades is of great significance, it has been seldom explored. In this work, the flow instability of a 16 straight-blade high-speed centrifugal pump is studied numerically at a rotational speed of $8500 \mathrm{rpm}$ and flow rate of $3 \mathrm{~m}^{3} / \mathrm{h}$. Results show that with the influence of rotor-stator interaction, time-domain pressure signals at the tongue show double peak characteristic, whereas a single peak characteristic exists at the diffuser wall. The pressure fluctuation near the tongue is reduced to approximately half of that at the volute wall by the water ring effect accompanied with the high-pressure factor. At the tongue region, the amplitude of the blade passing frequency is reduced by the unsteady flow, whereas the harmonic wave was increased at 2-4 times of the blade passing frequency.
\end{abstract}

\section{Introduction}

The high-speed centrifugal pump is prevalent in different applications with high head and compact structure, such as petrochemical, aero-engine, and oil refining equipment [1]. The operation request of centrifugal pump becomes stricter with the increment in the rotational speed of the centrifugal pump. The hydraulic interactions between the running blades and the stationery volute are well-known factors of vibrations and noises in the high-speed pump. The rotorstator interaction of the rotating impeller and the stationery volute could cause strong pressure pulsations and unsteady flow fields [2], which, in turn, may induce component fatigue, excessive noise, and vibration [3]. Therefore, the research on the unsteady flow of the high-speed pump by evaluating its transient characteristic and pressure fluctuation is of academic and social value.

Computational fluid dynamic simulations have been conducted to explore the rotor-stator interaction and flow features in high-speed pump in recent years. Alemi et al. [4] evaluated the design approach of the volute of the highspeed pump and its related performance and found that the behavior of the efficiency and radial force balance could be promoted by a design law to combine volute and radial diffuser hydraulically. Casimir et al. [5] numerically studied the rotor-stator interaction of a centrifugal pump at part load with special emphasis on the highly unsteady blade load. Jafarzadeh et al. [6] studied a pump with rotational speed of $13,000 \mathrm{rpm}$ and specific speed of 16 by using the numerical approach and concluded that the relative position of blades and the blade tongue has great effect on the stability and separation point of the flow. Yang et al. [7] studied a fullscale oxygen pump with rotational speed of $18,000 \mathrm{rpm}$ involved in a liquid rocket engine, investigated the clocking effect of the rotating parts, and found that the turbulent dissipation in the diffuser and the impeller directly leads to the clocking effect on the pump flow performance.

Despite the static performance of the high-speed pump, the dynamic characteristic of the rotating machinery has been studied by many researchers. Yang et al. [8] performed 


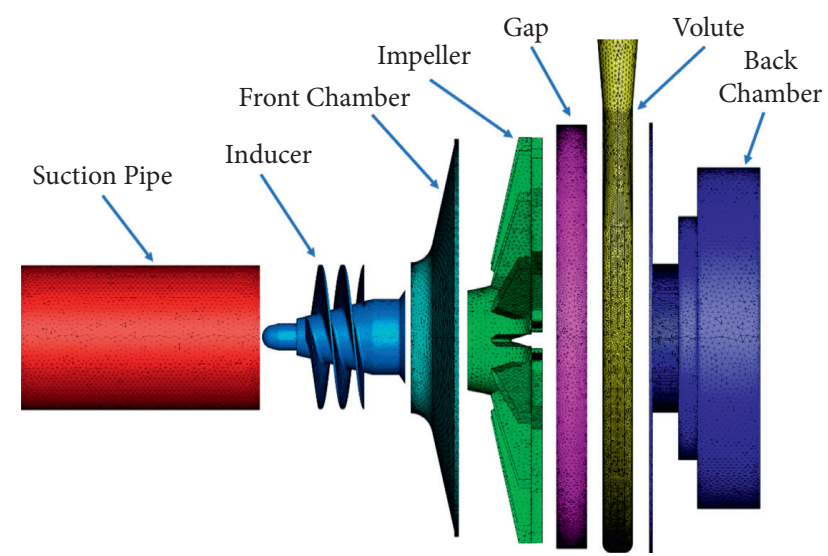

Figure 1: Computational domain and mesh of each part.

a computational study on the unsteady performance of a pump as turbine to find the optimal radial gap to achieve high efficiency. They found that the amplitude of the unsteady pressure pulsation in high frequency within the volute decreased with the increase in radial clearance, whereas that of the pressure pulsation in low frequency within the impeller remains unchanged. Xiao et al. [9] analyzed the main factors that affected the pressure fluctuations in a Francis pump as turbine. They found that the misaligned guide vanes reduce the amplitudes of the pressure pulse in low frequency in the draft tube by affecting the unsteady behavior of the vortex rope. Wang et al. [10] analyzed the vortex field and pressure fluctuation at multiple monitoring points in detail and concluded that abnormal amplitude increase in the frequency spectrums near the trailing edge strongly correlated with the evolution of vorticity.

Flow characteristics were analyzed to study the transient performance of high-speed pump within a certain blade structure. The effect of the splitter blade on the fluid performance of high-speed pumps has been studied well. Yuan et al. [11] evaluated the design principles of splitter blades, including deflection angle of blade and the number of blades. Yuan et al. [3] concluded that the spiral-profile blade inhibited the flow instability of the pump by affecting the pressure fluctuations more evenly.

The majority of the aforementioned studies are limited to the splitter blade. The study of straight blade, which has more difference when compared with spiral-profile blade, has been neglected [12]. With the design of the straight blade, one can produce high head, as well as low flow rate of a high-speed pump with cheaper and more handling-efficient pump structure design [13]. Given such characteristics, the highspeed pump with straight blade impeller fits in situations that require small flow rate, as well as high head, especially in smallscale pumps [14]. However, the high-speed pump with straight blade subjected to the interaction between the stationery volute and the rotational straight blade can lead to unsteady flow fields, especially those operating at off-design conditions. Rotor-stator interaction always leads to low efficiency. Su et al. [15] presented the flow stability analysis and entropy production to evaluate the regions of the hydraulic loss of straightblade high-speed pumps. Large hydraulic loss was observed at the trailing edge of the volute tongue and the blade. This phenomenon was explained by the large momentum exchange by flow separation and small region, including the sudden change in pressure gradients. Nevertheless, few studies on hydraulic performance and pressure pulsation of high-speed pumps with straight-profile blade were performed. The effect of rotor-stator interaction is important for the internal transient flow mechanism and pressure pulse performance of the highspeed pump with straight blade.

In this work, the hydraulic model of a high-speed centrifugal pump with 16 straight blades was established to numerically investigate the nonuniform distribution of flow in a straight-blade high-speed pump. The commercial software ANSYS-CFX was used to conduct the numerical study on the pressure pulse performance of the pump, and the numerical method was validated by performance experiments. The cause of the variation in the pressure fluctuation in different positions of the high-speed pump was investigated by analyzing the flow characteristics of the monitoring points.

\section{Materials and Methods}

2.1. Geometrical Model and Mesh Generation. With the designed rotation speed of $8500 \mathrm{r} / \mathrm{min}$, the 16-straight-blade high-speed pump was designed at a $250 \mathrm{~m}$ head and flow rate of $3 \mathrm{~m}^{3} / \mathrm{h}$. The computational domain and mesh generation of each part of the pump is shown in Figure 1. The detailed design parameters of the pump can be referred to in [15]. A test was conducted at the design condition to validate the independence of the grid of the high-speed pump. The computing deviation of the overall efficiency when the mesh numbers were in excess of 4,000,000 was less than $0.4 \%$. Therefore, the grid number adopted in the simulations was 4.28 million. The grids adjacent to the wall surfaces are specially treated, and the maximal $y+$ around the wall was below 100 to meet the computation requirements. The wall roughness was set as $1.3 \times 10^{-5} \mathrm{~m}$.

2.2. Solution Parameters. ANSYS-CFX 18.0 was adopted to perform the three-dimensional transient flow in the high-speed pump. The steady flow was first simulated, and its results were installed as the initial conditions for the transient flow to compute the pump pressure pulsations in the pump. The transient rotor-stator interface was adopted in the transient state simulation. The shear stress transport (SST) $k$ - $\omega$ turbulence model with scalable wall functions was used because of its good accuracy in rotary flow computation. A second-order accuracy algorithm was selected for all temporal and spatial derivatives. Table 1 summarizes the boundary conditions adopted in the computation. The wall surface roughness was set as $25 \mu \mathrm{m}$. The turbulent intensity at the inlet boundary is set as $5 \%$. The convergence criterion of the mass and momentum was $10^{-6}$. Figure 2 shows the monitoring points mounted on the volute and impeller in the pump. One cycle spends $0.00706 \mathrm{~s}$, and the total time was set to $0.0706 \mathrm{~s}$ to ensure 10 rounds of unsteady simulation data.

Pressure pulsation is one of the crucial reasons of vibration and noise in high-speed centrifugal pump. A total of 
TABLE 1: Boundary conditions adopted in the numerical simulation.

\begin{tabular}{lc}
\hline Location & Boundary condition \\
\hline Inlet duct & Velocity inlet \\
Outlet duct & Pressure outlet \\
Stationary surfaces & No-slip wall \\
Connection interfaces & Transient rotor-stator \\
\hline
\end{tabular}

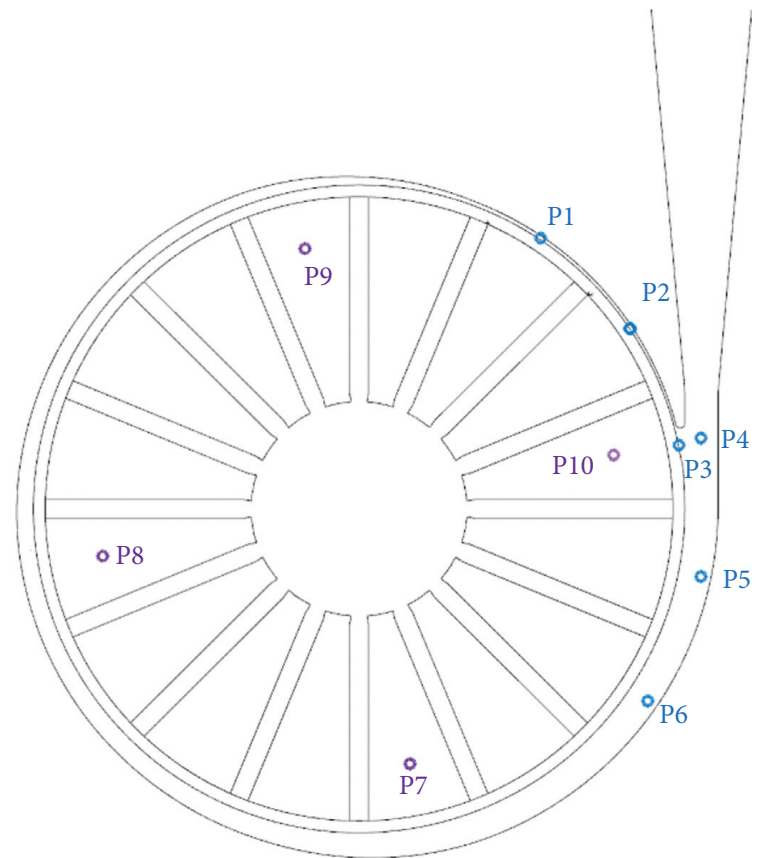

FIgURe 2: Pressure pulsation monitoring point settings $(P-1-P-6$, volute and tongue; $P-7-P-10$, impeller passage).

10 monitoring points mounted at the central plane of the impeller and volute are available to capture time-amplitude and amplitude-frequency characteristics (Figure 2). The calculation lasted for 10 impeller revolution periods (total period), and the calculation of frequency domain was conducted from the last five impeller revolution periods via fast Fourier transform (FFT) method.

2.3. Experiment Validation. The hydraulic performance test of the pump was carried out at the Key Laboratory of Fluid Transmission Technology of Zhejiang Province, Zhejiang Sci-Tech University. Figure 3 shows the test rig of the experiment, which mainly comprises a test pump, a flowmeter, two pressure transmitters, and several regulating valves and pipelines. Room-temperature water was used as the working fluid. The pump inlet and outlet pressures and the flow rates were measured with the accuracy of $0.25 \%$ and $0.2 \%$, respectively. The head, flow rate, power, and efficiency were measured by the specific sensors with uncertainty of $\pm 0.895 \%, \pm 0.55 \%, \pm 0.956 \%$, and $\pm 1.421 \%$, respectively [15] .

Figure 4 provides the comparison of the external characteristics of experiments and numerical prediction at different flow rates. Generally, the agreement between the numerical predictions and experimental data was shown

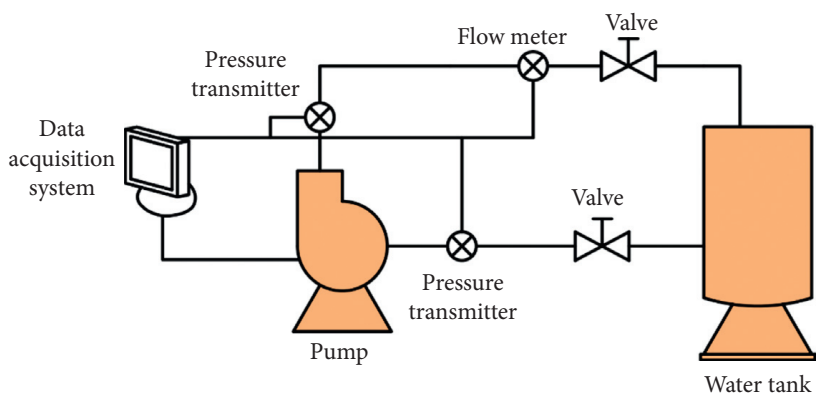

FIgURE 3: Schematic of experimental apparatus.

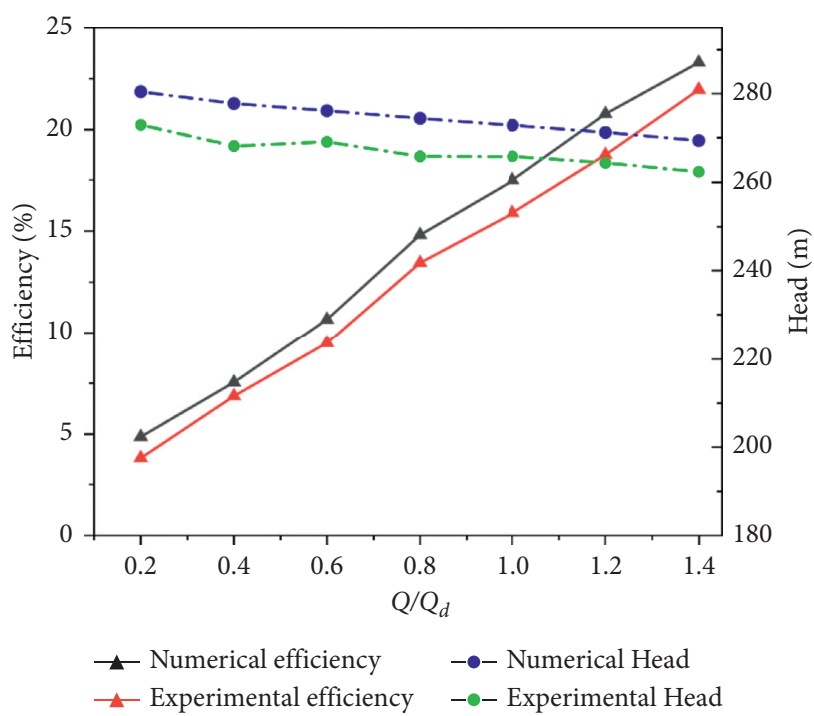

FIGURE 4: Comparison of experiment and simulation.

with a relative error that is less than $5 \%$. On account of neglect of the seal and mechanical losses in the numerical method, simulation predictions are slightly larger than those of the experiment data.

\section{Results and Discussion}

The unsteady pressure fields of the high-speed pump with flow rates of 0.2 design flow rate $\left(Q_{d}\right), 0.4 Q_{d}, 0.6 Q_{d}, 0.8 Q_{d}$, and 1.0 $Q_{d}$ were conducted to study and analyze the flow characteristics and unsteady pressure pulsations within the volute and the impeller.

3.1. Flow Characteristics. Dimensionless quantities are used in this paper for the convenience of the comparison of flow characteristics in different flow rates, as well as the pressure pulsation analysis in time and frequency domains. The dimensionless pressure coefficient $C_{p}$ is defined as follows:

$$
C_{p}=\frac{P-P_{\text {inlet }}}{0.5 \rho v_{u}^{2}}
$$

where $P$ is the static pressure, $P_{0}$ is the static pressure at a sampling point, $\rho$ is the fluid density, and $v_{u}$ is the circumferential velocity at the outer edge of the impeller. 
The dimensionless velocity $C_{v}$ is defined as follows:

$$
C_{v}=\frac{v}{v_{u}} .
$$

Moreover, the dimensionless pressure gradient is defined as $\partial P / \partial L=\Delta P^{*} / R$, where $R$ is the radius of the impeller. In the analysis of pressure fluctuation, the dimensionless pressure coefficient $C_{p f}$ is defined as follows:

$$
\begin{aligned}
C_{p f} & =\frac{P-\bar{P}}{0.5 \rho v_{u}^{2}}, \\
\bar{P} & =\frac{P-\bar{P}}{0.5 \rho v_{u}^{2}} \sum_{i=0}^{N-1} p\left(t_{i}\right) .
\end{aligned}
$$

Figure 5 shows the pressure field in the central section of the impeller under different flow rates. The monitoring points near the volute were marked in the figure. The fluid pressure in the impeller increases from the inlet to the outlet in the rotation operation.

At $0.2 Q_{d}$, when the blade rotates close to the tongue, the near-tongue region exhibits a local high-pressure area because of the squeezing action of the blade. Apparent squeezing action leads to higher pressure fluctuation in $0.2 Q_{d}$ than those in 1.0 $Q_{d}$ and 1.4 $Q_{d}$ (Figure 6). The fluid with high flow rate that goes through the tongue may lead to unapparent squeezing phenomenon in $1.0 Q_{d}$ and $1.4 Q_{d}$. The resistance force may play a negative role in improving the steady flow in the high-speed centrifugal pump according to the decrease in the pulsating pressure amplitude. The pressure and valley value of pressure pulsation in the tongue area stays high during peak periods, thereby suggesting the small pressure fluctuation at the monitoring points in the tongue area. The quantity analysis of the pressure fluctuation can be referred to in Table 2 .

To clearly show the characteristics, the pressure gradient in $0.2 Q_{d}$ is illustrated in Figure 7. The area with high-pressure gradient (red region) was located at the near-tongue region and changes slightly with the impeller rotation. A small highpressure gradient area (red area) is dispersedly distributed at the pressure side of the trailing edge and synchronized with blade motion. The pressure gradient of the area swept by the blade trailing edge will continuously fall back to and then recover from the peak, thereby resulting in pressure pulsation with the blade passing frequency $\left(f_{B P F}\right)$. With the increment in flow rates, the near-tongue region with high-pressure gradient increases, but differences on the overall distribution are lesser (not shown). The high-pressure gradient area formed at the pressure side of the trailing edge is an important reason for the pressure pulsation on the rotor-stator interface of centrifugal pumps.

At the same impeller position in the radial direction, the pressure on the pressure surface of the blade is lower than that on the suction surface (Figure 5). When analyzing the streamlines at 1.0 $Q_{d}$ at the time of pressure pulsation valley (Figure 8), the flow is noted in poor guidance along the impeller passages. The circumferential sliding fluid is mixed with the radial entering fluid and then formed recirculation vortices because of the $90^{\circ}$ leading edge of the straight blade and the pressure difference between the pressure and suction surface. The counter-clockwise vortices were strengthened after the flow passage has swept over the tongue because the narrow space restricts the fluid that flows in the volute. Furthermore, although the volutes at Points 21 and 22 are much wider, the recirculation vortex also exists. The high pressure at the near-tongue region restricts the fluid that enters into the divergent suction. The high pressure may also reduce the pressure fluctuation at the near-tongue region. At $0.2 Q_{d}, 0.6 Q_{d}$, and $1.4 Q_{d}$, the overall trend of counterclockwise vortices in the certain passages remain unchanged (not shown).

The distribution of turbulence kinetic energy was simulated to study the turbulent flow in the pump (Figure 9). Large turbulence kinetic energy is observed at the area between the larynx of the volute and the blade tips. Large turbulence kinetic energy increased as the blade tip rotates with respect to the near-tongue region (Figures 9(a) and 9(b)). Turbulence kinetic energy does not display apparent difference in the pressure or suction surface of the blade. Another area with large turbulence kinetic energy was observed in the diffuser because of the backflow in the diffuser (as shown in the streamline in Figure 8), thereby suggesting that the diffuser should be designed with a larger diameter.

3.2. Flow Fluctuation. Figure 6 shows the time domain of the transient pressure at the two monitoring points at the neartongue region. The fluctuated pressure in a cycle of the impeller rotation experiences a periodical change. At certain flow rates, different phenomena of periodic pressure variations between Points 3 and 4 were observed. The double peak characteristic of Point 3 is caused by the rotor-stator interaction between a straight blade and a volute. At certain point, the fluctuating amplitudes reduced from $0.2 Q_{d}$ to 1.0 $Q_{d}$ and increased slightly to $1.4 Q_{d}$ because high flow rate caused high pressure at the near-tongue region and was less influenced by the effect of the rotor-stator interaction. In Point 4 , the pressure pulsation fluctuates as a single peak characteristic with the amplitude almost the same as that in Point 3.

Figure 10 shows the curve of pressure pulsation at the monitoring points at the diffuser about to or just swept over the tongue area. At a certain flow rate, similar periodic pressure variations are observed for the four points. At the upstream points (e.g., $P-22$ and $P$-21), the fluctuating amplitudes decreased from $0.2 Q_{d}$ to $1.0 Q_{d}$ and increased slightly to $1.4 Q_{d}$. Meanwhile, at the downstream points (e.g., $P-35$ and $P-34)$, the pressure exhibits a monotonic reduction with the increase in the flow rate.

Table 2 lists the peak-to-peak amplitude values of the pressure pulsations of the six monitoring points at their main frequencies. The amplitudes of Points 3 and 4 are approximately half of that at most of the points in the volute wall because the high pressure at the narrow near-tongue region reduced the pressure fluctuation.

The frequency domain of pressure at the near-tongue region is shown in Figure 11 . The shaft rotating frequency $\left(f_{s}\right)$ based on the impeller rotating speed of $8500 \mathrm{r} / \mathrm{min}$ is $141.67 \mathrm{~Hz}$. Another important frequency, 


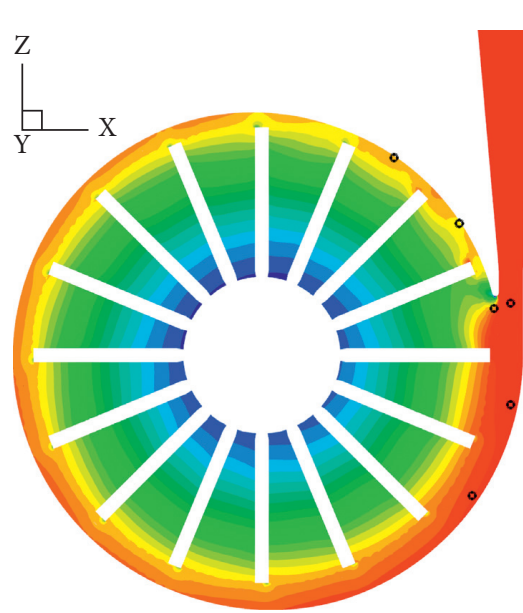

(a)

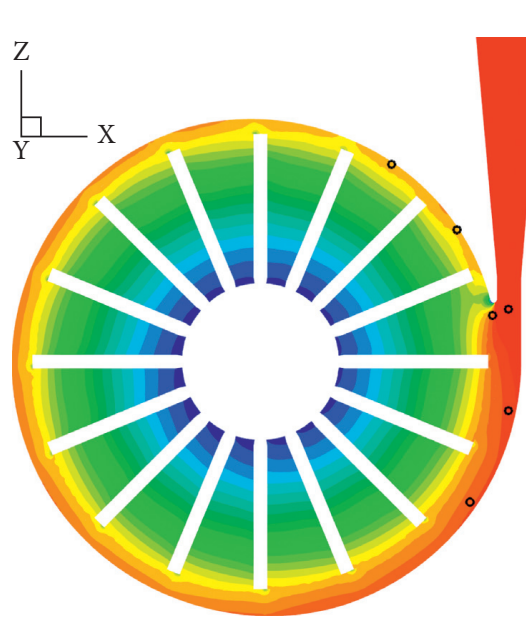

(c)

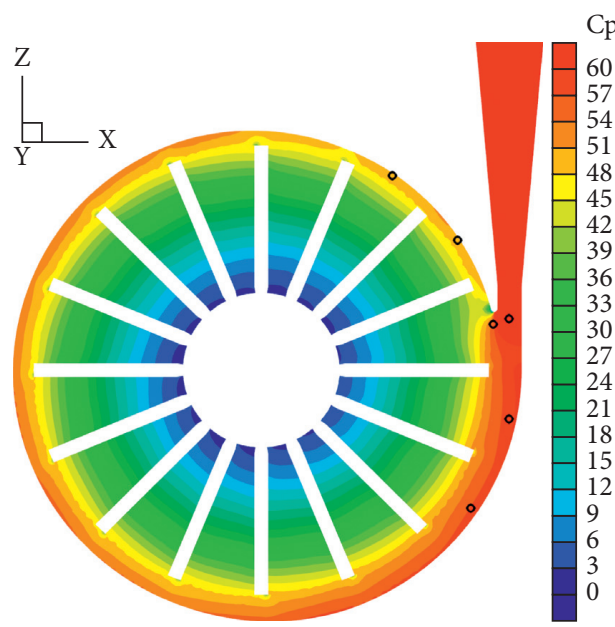

(e)

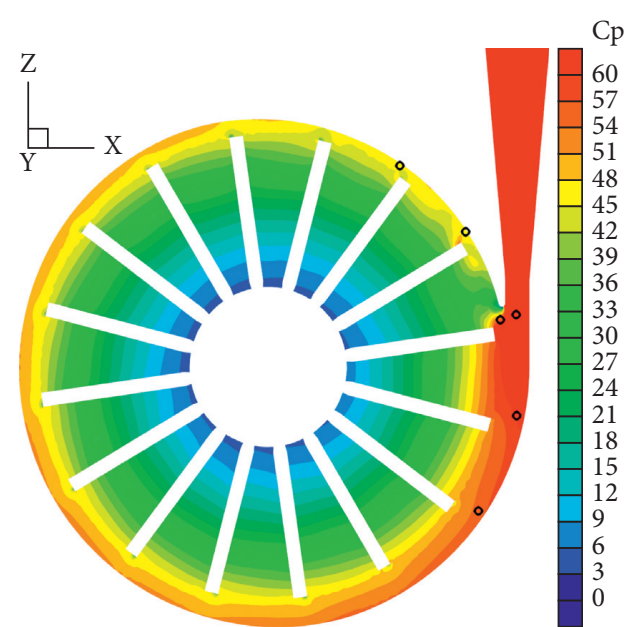

(b)

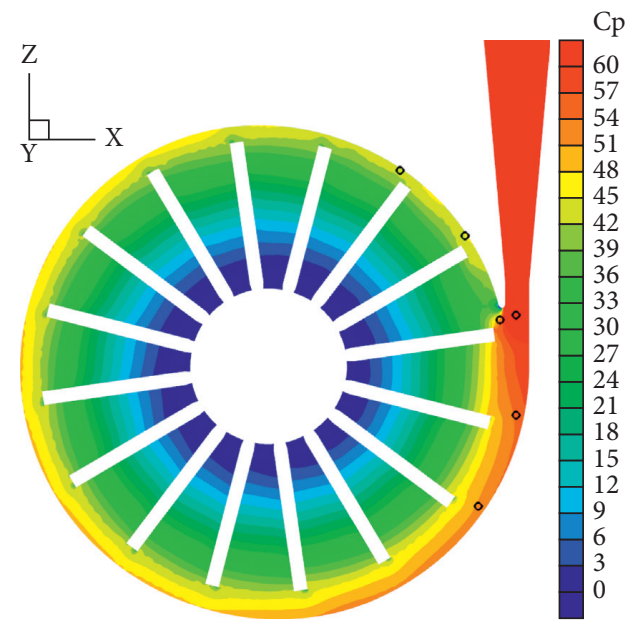

(d)

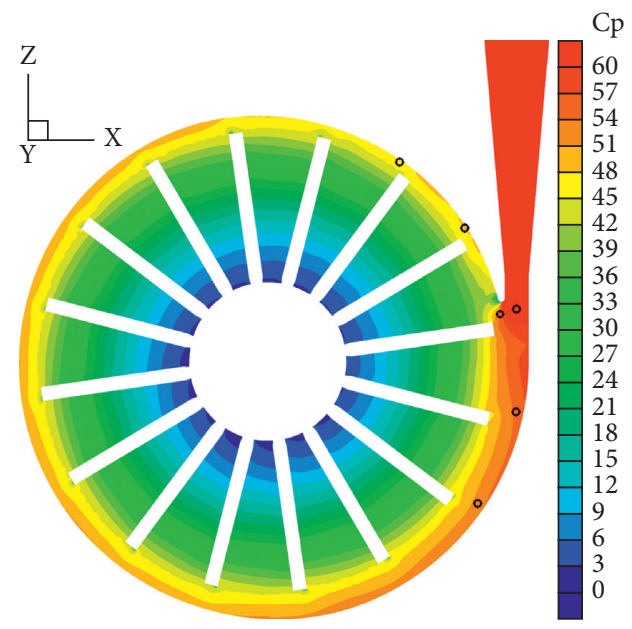

(f)

FIGURE 5: Distribution of static pressure in the central section of impeller under different flow rates: (a) $0.2 Q_{d}$, at pressure pulsation peak; (b) $0.2 Q_{d}$, at pressure pulsation valley; (c) $1.0 Q_{d}$, at pressure pulsation peak; (d) $1.0 Q_{d}$, at pressure pulsation valley; (e) $1.4 Q_{d}$, at pressure pulsation peak; and (f) $1.4 Q_{d}$, at pressure pulsation valley. 


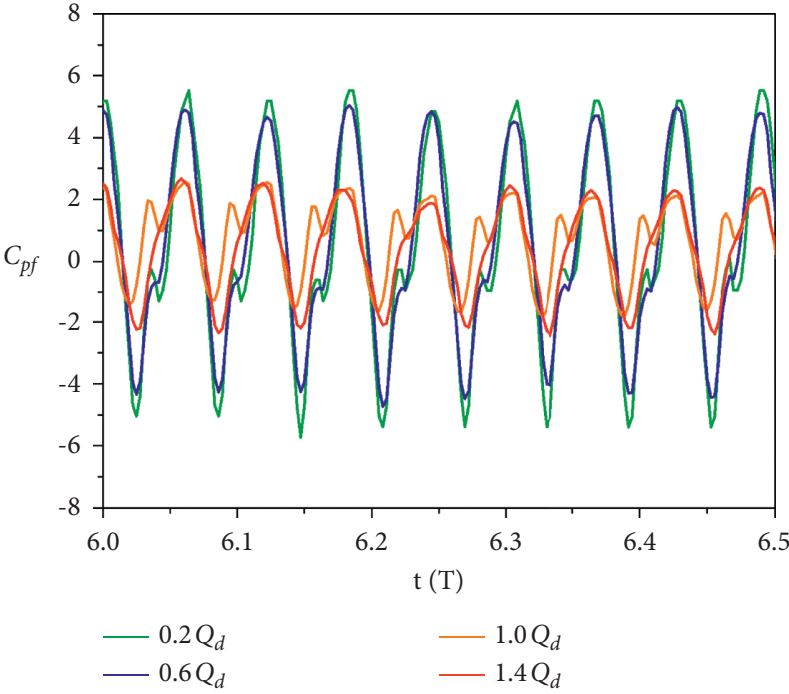

(a)

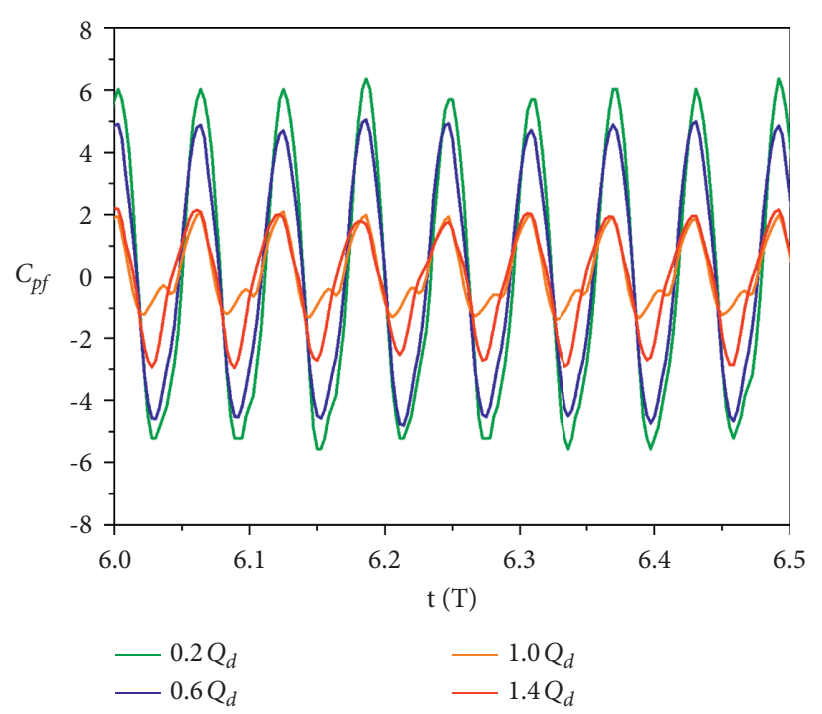

(b)

FIGURE 6: Time-domain pressure signals of the tongue area with different flow rates for monitors: (a) 3 and (b) 4.

TABLE 2: Amplitude of pressure fluctuation of points at the volute.

\begin{tabular}{lcccc}
\hline Monitoring point & \multicolumn{3}{c}{ Amplitude (MPa) } \\
\hline 1 & $0.2 Q_{d}$ & $0.6 Q_{d}$ & $1.0 Q_{d}$ & 0.21 \\
2 & 0.65 & 0.46 & 0.21 & 0.21 \\
3 & 0.65 & 0.48 & 0.11 & 0.21 \\
4 & 0.31 & 0.23 & 0.09 & 0.15 \\
5 & 0.33 & 0.28 & 0.12 & 0.24 \\
6 & 0.53 & 0.44 & 0.18 & 0.28 \\
\hline
\end{tabular}

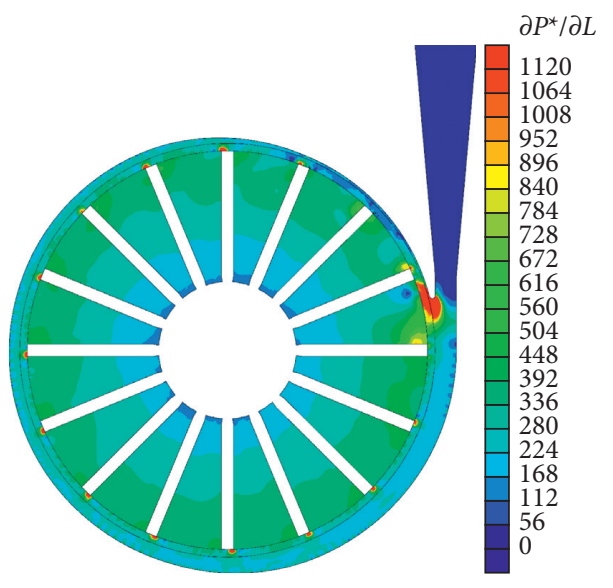

(a)

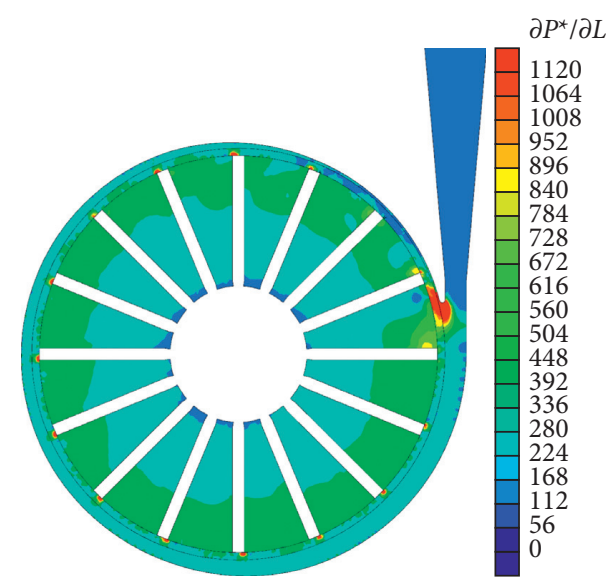

(b)

Figure 7: Distribution of static pressure in the central section of the impeller under the flow rate of $0.2 Q_{d}(\mathrm{a})$ at pressure pulsation peak and (b) at pressure pulsation valley.

$f_{B P F}$, is $2266.67 \mathrm{~Hz}$ on account of the impeller number of 16. As shown in Figure 11, the maximum pressure amplitude occurs at $f_{B P F}$. The main frequency at the near-tongue region is the rotating frequency, which plays a decisive role in the pressure pulsation of the pump. The high-frequency pulsation at $f_{B P F}$ is $2-4$ times larger in Point 3 than that in Point 4, thereby illustrating that fluctuations at the tongue caused by unsteady flow reduced the effect of the impeller and formed the harmonic wave of $f_{B P F}$. 


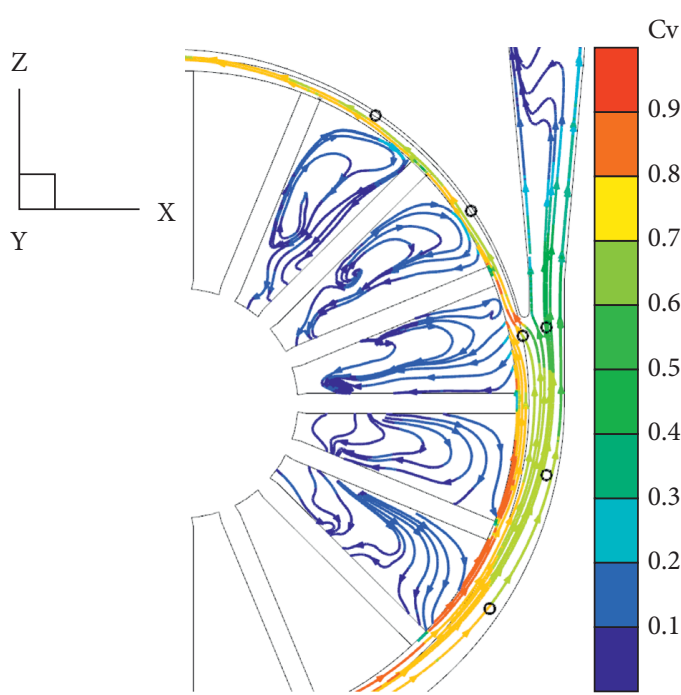

(a)

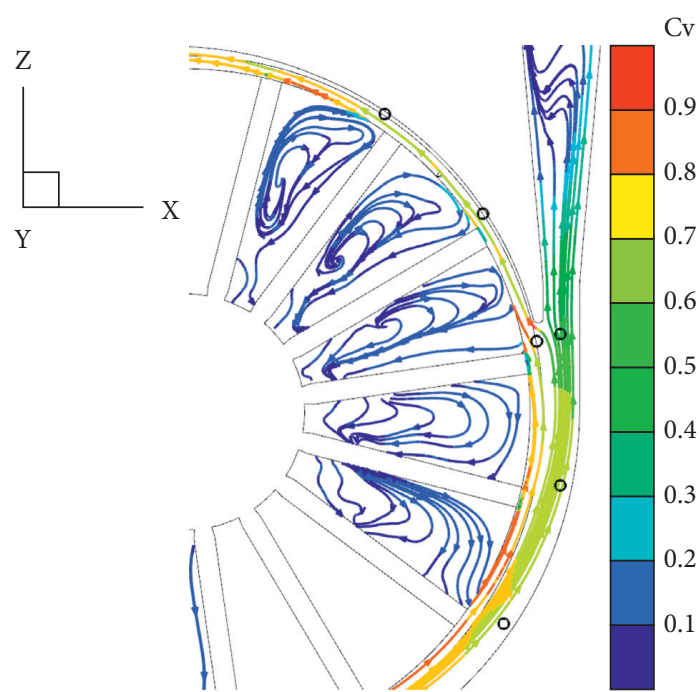

(b)

FIGURE 8: Streamline distribution of relative velocity in the central section of the impeller and the volute: (a) $1.0 Q_{d}$ at pressure pulsation peak and (b) $1.0 Q_{d}$ at the pressure pulsation valley.

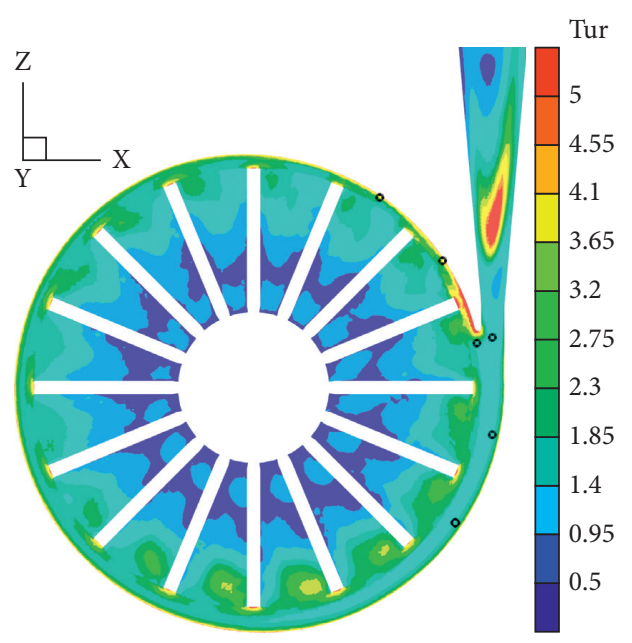

(a)

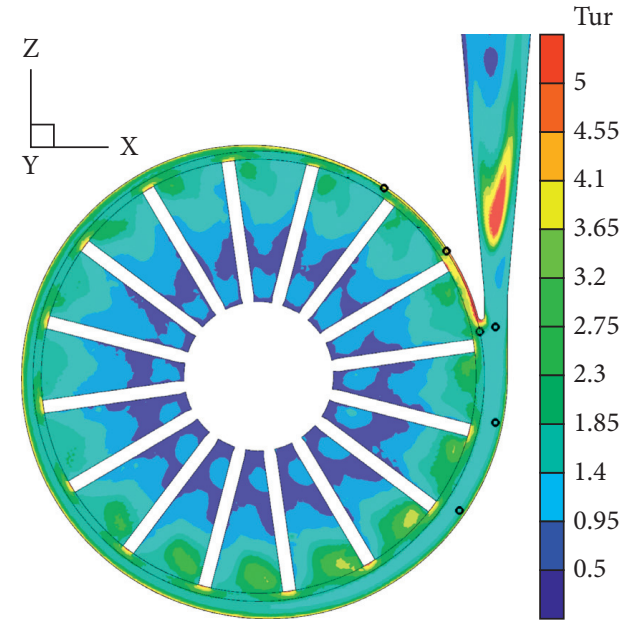

(b)

FIGURE 9: Distribution of turbulence kinetic energy in the central section of the impeller at $1.0 Q_{d}$ (a) at pressure pulsation peak and (b) at pressure pulsation valley.

Figure 12 shows the frequency domain of pressure at the volute not far from the near-tongue region. The transient pressure pulsation within the impeller corresponds to the composition of the transient pressure pulsation caused by the impeller-volute interaction. The propagation of unsteady pressure from the volute to the impeller is influenced by the flow rate. In total, the flow rate has a negative effect on the performance of the high-speed centrifugal pump.

The amplitude of the pressure pulsations at two frequencies of $f_{s}$ and $f_{B P F}$ is listed in Table 3 . In the volute, the shaft rotating frequency is lesser than the main frequency $f_{B P F}$. Combined with the pressure contour (Figure 5), the low amplitude of pressure fluctuation at Point 36 is possibly due to the water ring effect [16]. The rotating impeller provides the fluid with large tangential velocity, thereby forming a water ring between the volute and the impeller tip, which may reduce part of the rotor-stator interaction to the pressure fluctuation at $16 f_{s}$. Figures 8 and 9 show that backflow phenomenon exists in the exit part of the pump, indicating that the exit part was designed to be narrow. Narrowed diffuser leads to high pressure at the near-tongue region. The influence of the water ring at high-speed accompanied with the effect of narrow diffuser reduced the pressure fluctuation at Point 3. The deviation from the design flow rate may reduce the water ring effect, thereby increasing pressure fluctuation. Influenced by the tongue region represented by Point 3 , the pressure fluctuation on Point 4 shows similar laws. 

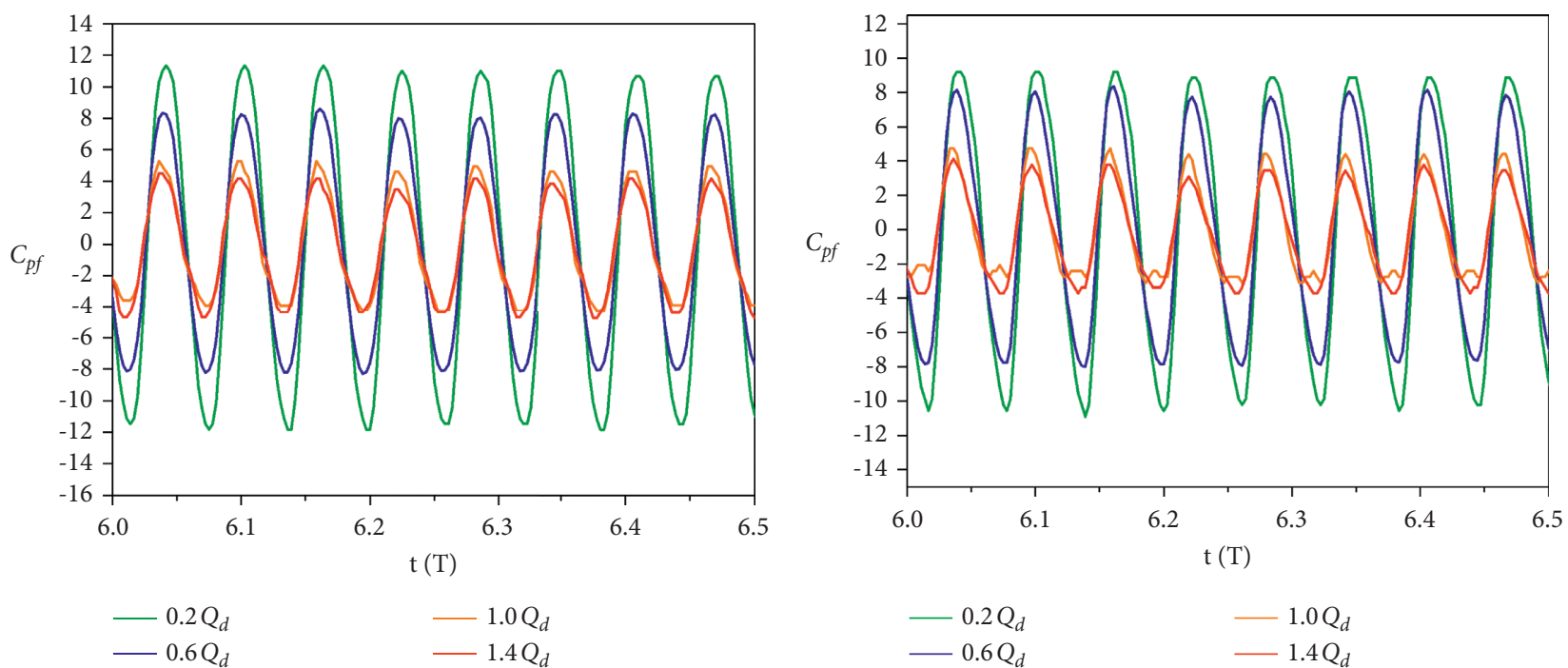

(a)

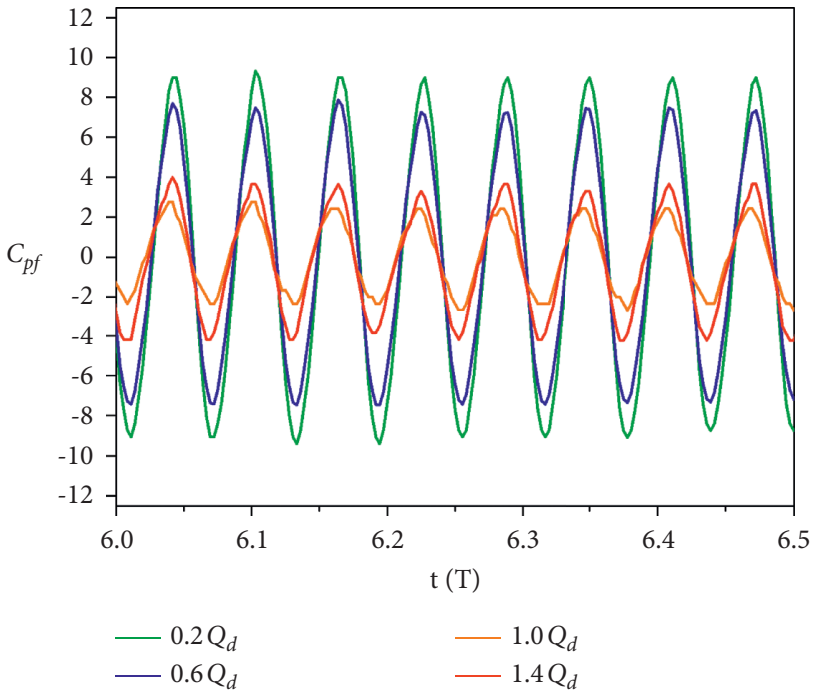

(c)

(b)

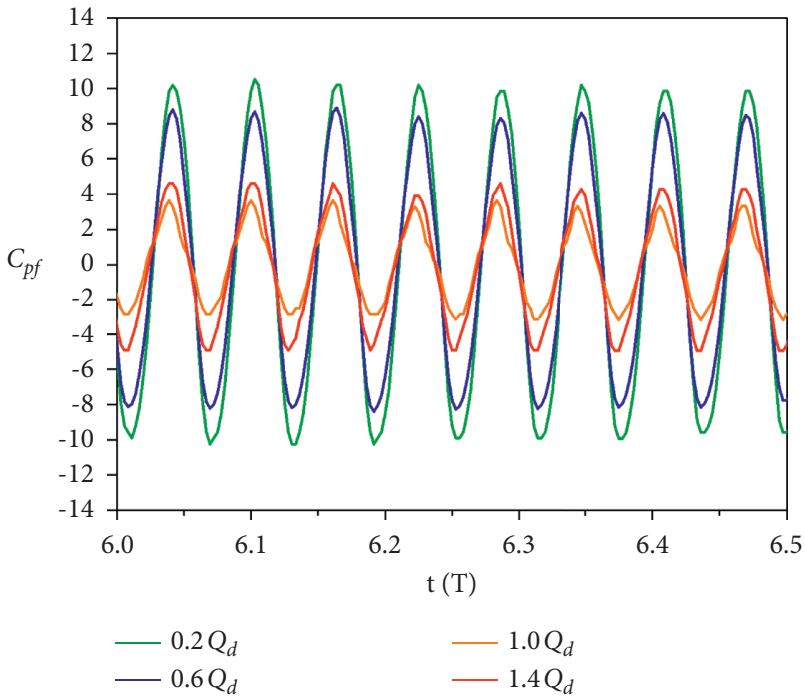

(d)

Figure 10: Time-domain pressure signals of the diffuser at the upstream and downstream of the tongue area with different flow rates for monitoring points: (a) 1, (b) 2, (c) 5, and (d) 6 .

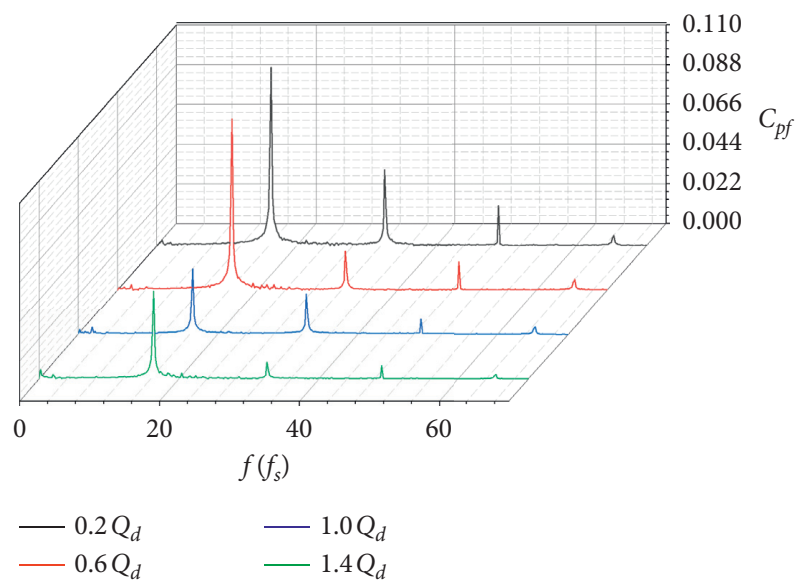

(a)

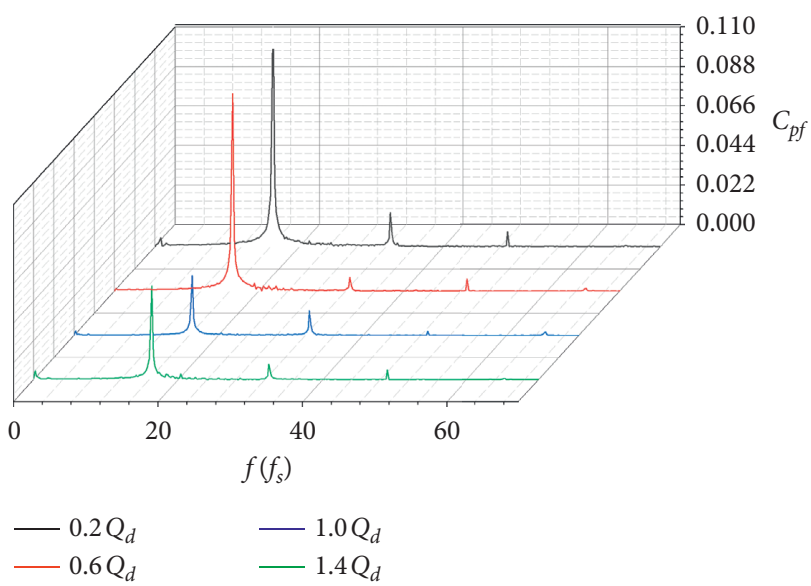

(b)

Figure 11: Pressure spectra at the tongue area for monitors: (a) 3 and (b) 4 . 


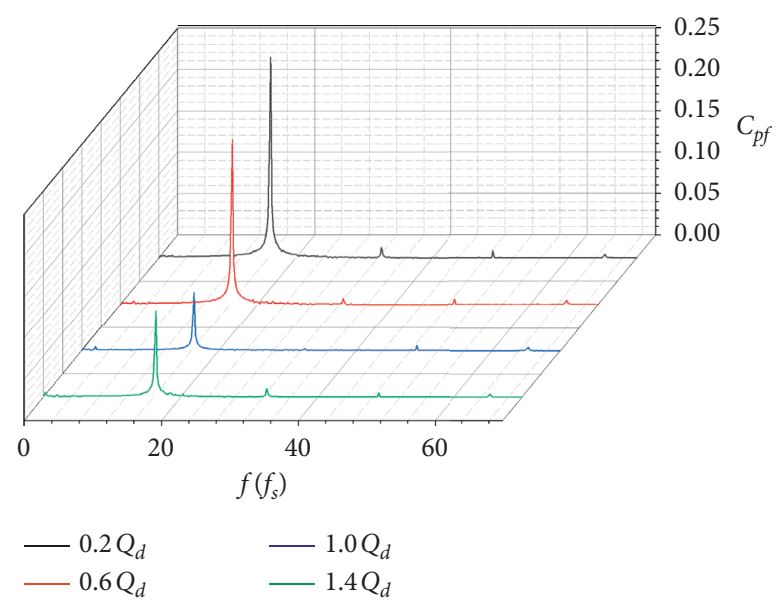

(a)

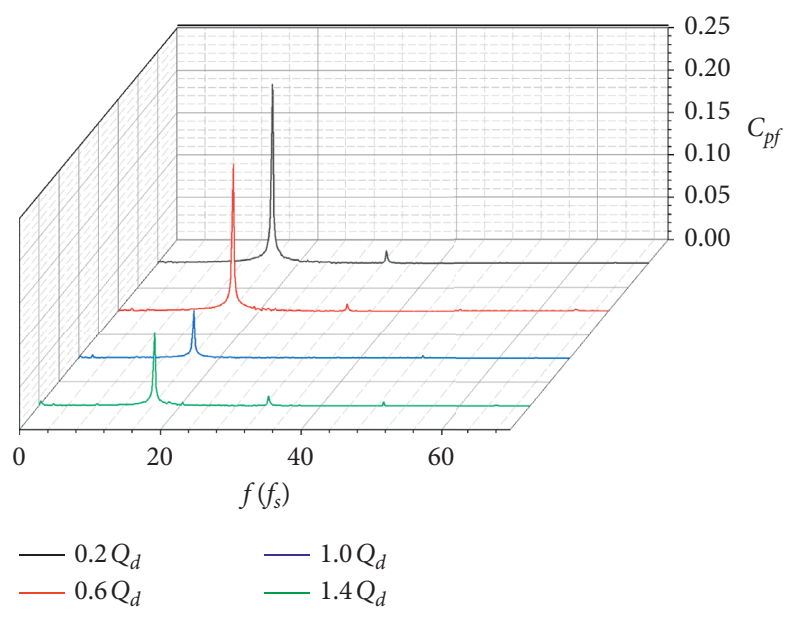

(c)

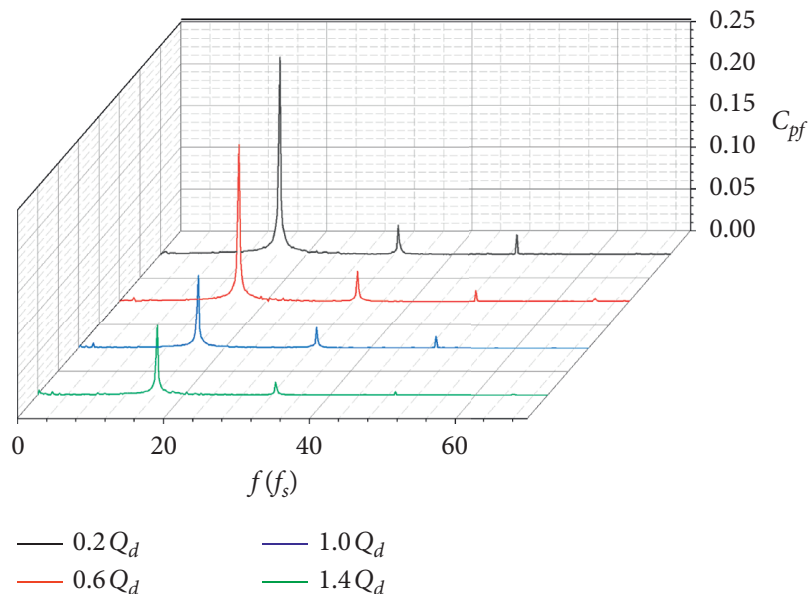

(b)

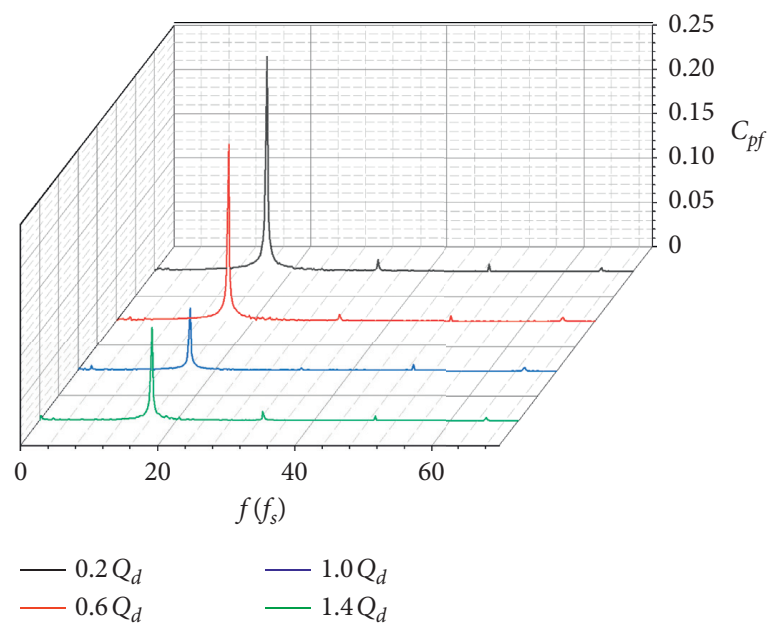

(d)

Figure 12: Pressure spectra of the diffuser for monitoring points: (a) 1, (b) 2, (c) 5, and (d) 6 .

TABLE 3: Amplitude of the main frequency at the impeller monitors.

\begin{tabular}{|c|c|c|c|c|c|c|c|c|}
\hline \multirow{2}{*}{$Q / Q_{d}$} & \multirow{2}{*}{ Frequency $(\mathrm{Hz})$} & \multicolumn{7}{|c|}{ Amplitude $(\mathrm{kPa})$} \\
\hline & & 35 & 34 & 22 & 21 & 32 & 36 & 37 \\
\hline \multirow[t]{2}{*}{0.2} & 141.67 & 1.71 & 2.28 & 2.44 & 2.08 & 2.42 & 1.02 & 0.0005 \\
\hline & 2266.67 & 235.24 & 278.84 & 242.57 & 211.07 & 273.52 & 98.9 & 134.63 \\
\hline \multirow[t]{2}{*}{0.6} & 141.67 & 2.20 & 2.23 & 2.55 & 2.14 & 2.54 & 1.52 & 0.0008 \\
\hline & 2266.67 & 186.92 & 200.69 & 199.61 & 173.01 & 212.17 & 94.89 & 111.15 \\
\hline \multirow[t]{2}{*}{1.0} & 141.67 & 2.15 & 2.13 & 1.61 & 1.33 & 1.85 & 1.30 & 0.0004 \\
\hline & 2266.67 & 86.98 & 108.99 & 70.68 & 57.2 & 99.93 & 36.41 & 33.24 \\
\hline \multirow[t]{2}{*}{1.4} & 141.67 & 1.91 & 1.78 & 1.78 & 1.21 & 1.44 & 1.35 & 0.0005 \\
\hline & 2266.67 & 83.25 & 101.13 & 104.46 & 85.4 & 107.37 & 48.96 & 52.29 \\
\hline
\end{tabular}

To further discuss the pressure fluctuation in the highspeed pump, we also monitor the transient pressure fields in the impeller. Figure 13 shows the pressure fluctuation curve of the four points at the impeller passage in the interval of $90^{\circ}$ in the circumferential direction in one rotational cycle. The pressure fluctuates violently under small flow conditions, and the harmonics of the multiplication of shaft rotating frequency exist. The periodic characteristic of pressure fluctuation in the impeller is more complex than that in the volute. Figure 13 certifies that the rotor-stator interaction of the rotating impeller and the stationary volute would cause an unsteady pressure pulsation from the volute and within the impeller with the main frequencies of $f_{s}$ and $f_{B P F}[11]$.

Figure 14 shows the frequency spectrum of the monitoring points in the impeller. The amplitude of $f_{B P F}$, which plays a decisive role in the pressure fluctuation of the impeller, is far greater than those of other frequencies. The amplitude of the main frequency is affected by the flow 

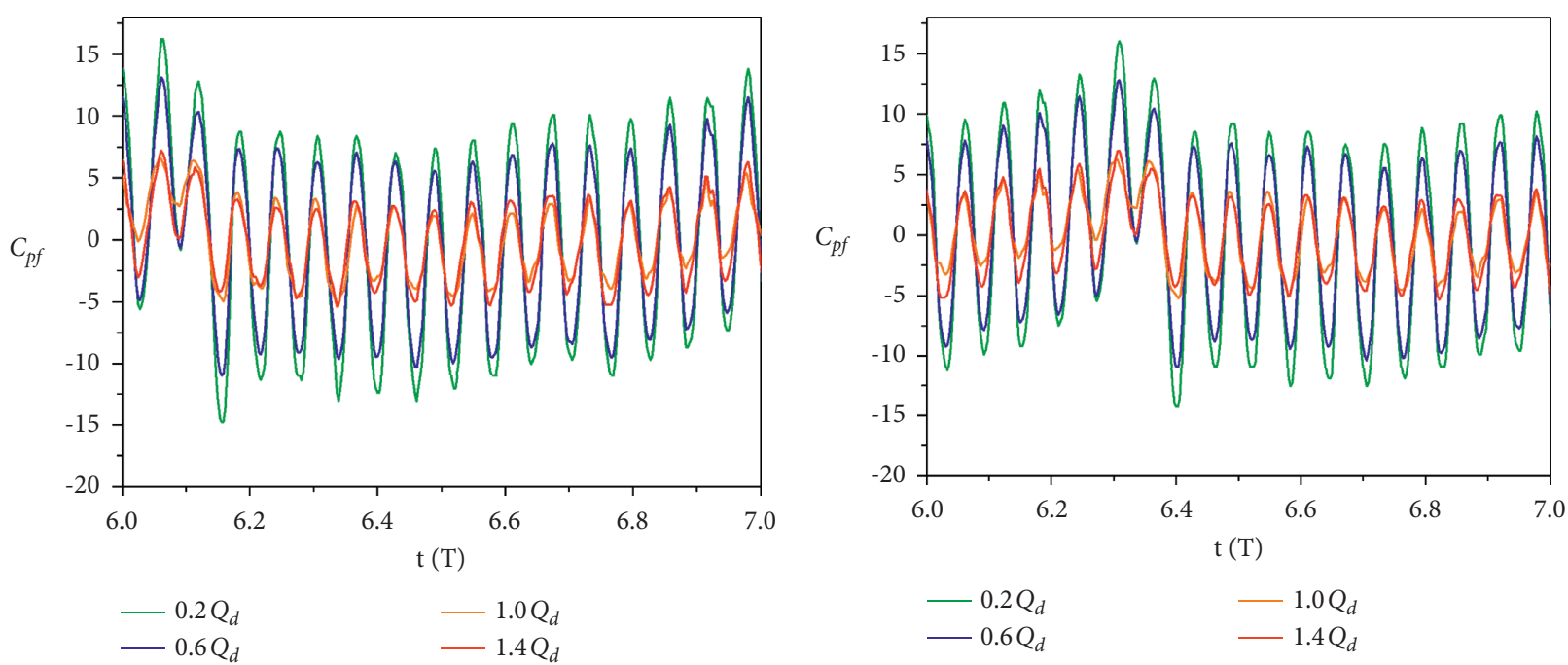

(a)

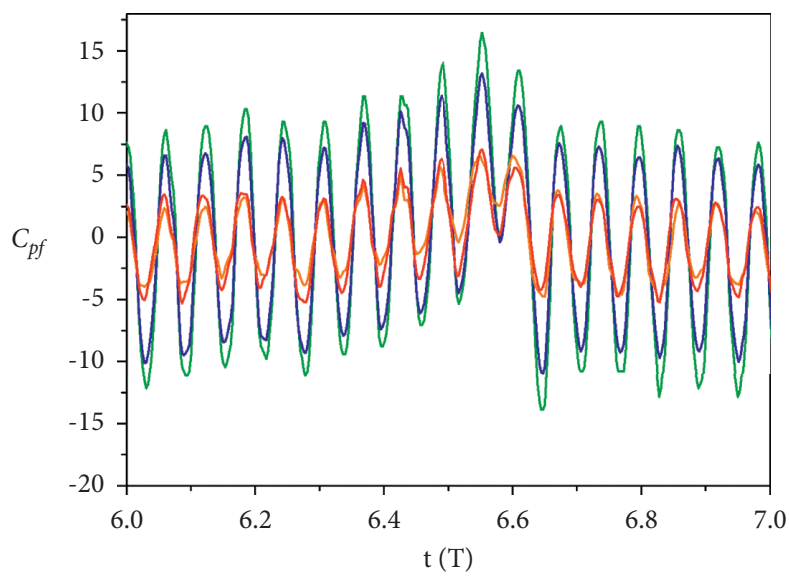

(b)

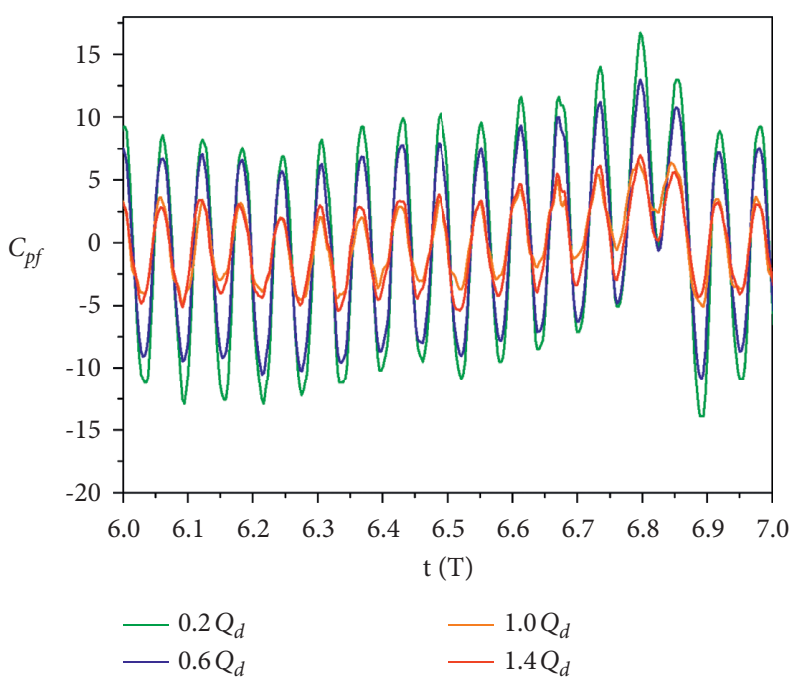

(c)

(d)

FiguRe 13: Pressure signals at the time domain of the diffuser at the impeller with different flow rates for monitoring points: (a) 7, (b) 8, (c) 9, and (d) 10 .

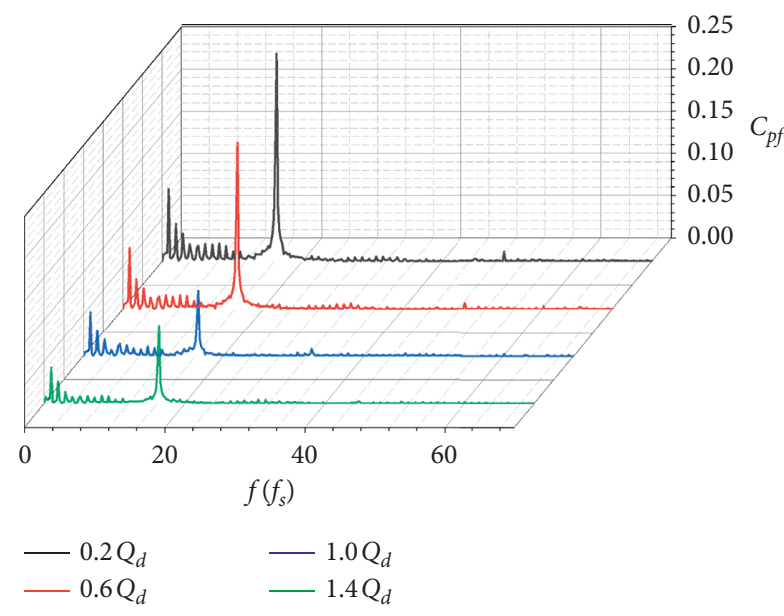

(a)

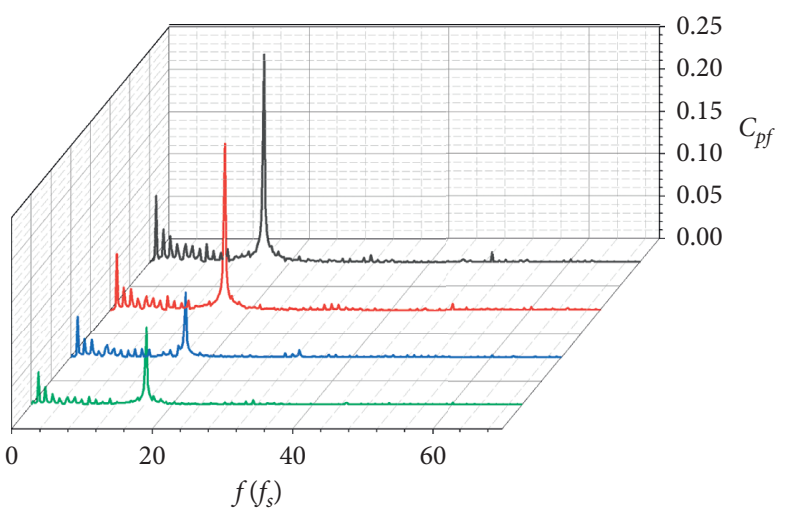

$\begin{array}{ll}-0.2 Q_{d} & -1.0 Q_{d} \\ -0.6 Q_{d} & -1.4 Q_{d}\end{array}$

(b)

Figure 14: Continued. 


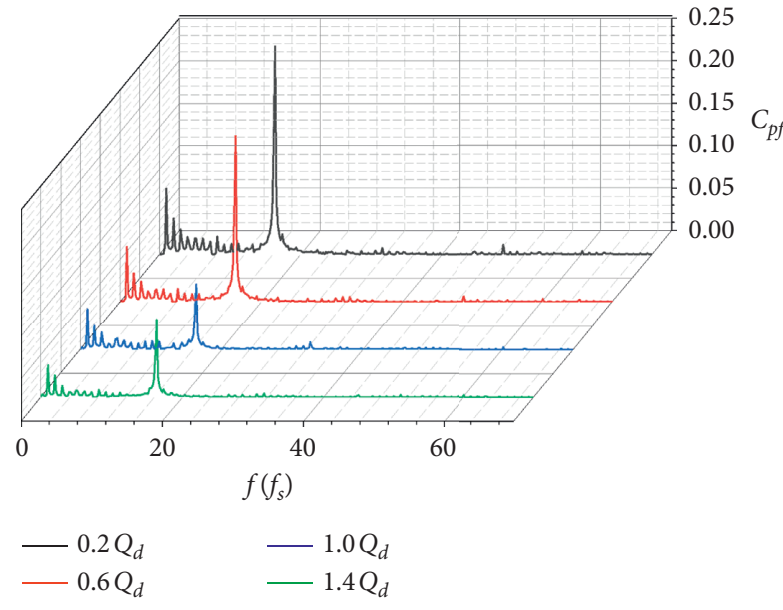

(c)

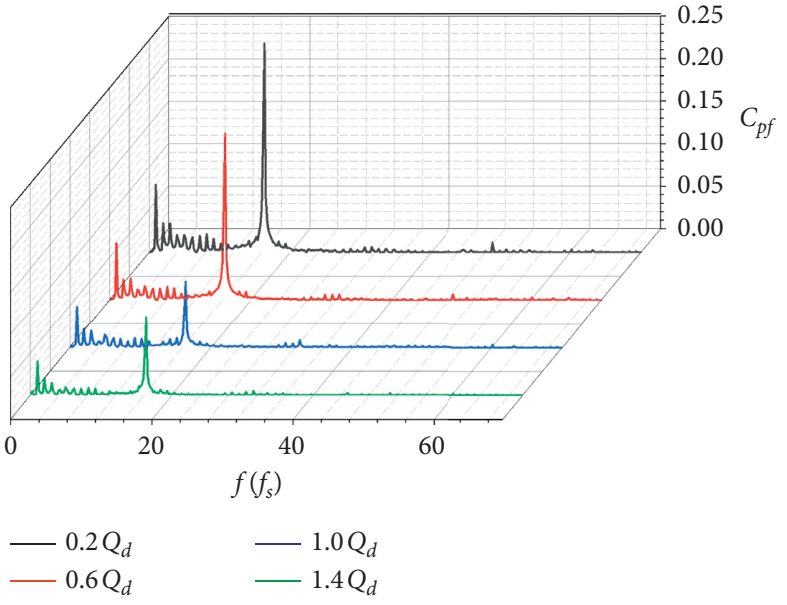

(d)

Figure 14: Pressure spectra of the diffuser for monitoring points: (a) 7, (b) 8, (c) 9, and (d) 10.

condition. In general, the smaller the flow is, the larger the amplitude of the main frequency will be. Compared with other points, the main frequency amplitude at the tongue is obviously reduced and is consistent with that in the volute. The main reason is that the low-frequency pulsation caused by the unsteady flow is dominant under the small flow conditions. The fluctuation tendency does not exhibit apparent differences among various points because the monitoring points are far away from the volute.

\section{Conclusions}

A procedure has been presented to numerically investigate the flow characteristics and pressure fluctuation of a high-speed pump with straight blades. The type of highspeed pump used in this manuscript can be used in the application of petrochemical industry, catalyst feeding, etc., in which the working medium to be pressurized through the pump is kept in low flow rate so as to enter the certain device. The main conclusions are summarized as follows:

(1) Owing to the rotor-stator interaction between the straight blade and the volute, the time-domain pressure signals at the tongue show double peak characteristic, whereas the transient pressure at the diffuser wall shows single peak characteristic.

(2) The high-pressure gradient area formed at the pressure side of the trailing edge is an important factor that affects pressure pulsations on the rotorstator interface of the centrifugal pumps.

(3) With the narrowly designed tongue structure, the high pressure at the near-tongue region restricts the fluid from entering into the divergent suction. The water ring effect combined with the high-pressure factor reduces the pressure fluctuation at the neartongue region to approximately half of that at the volute wall.
(4) In the tongue region, the unsteady flow reduces the amplitude of the blade passing frequency and increases the harmonic wave by $2-4$ times that of the blade passing frequency.

\section{Data Availability}

The data used to support the findings of this study are included within the article.

\section{Conflicts of Interest}

The authors declare no conflicts of interest.

\section{Acknowledgments}

This work was supported by the National Nature Science Foundation of China (52071296 and 51706206), Natural Science Foundation of Zhejiang Province (LZ15E090002 and LGF19E060001), Science Foundation of Zhejiang SciTech University (ZSTU) (19022101-Y), Top-Notch Talent Support Program of Zhejiang Province (2019R51002), and Key Research and Development Program of Zhejiang Province (2020C01027).

\section{References}

[1] X. Jia, B. Cui, Z. Zhu, and X. Yu, "Numerical investigation of pressure distribution in a low specific speed centrifugal pump," Journal of Thermal Science, vol. 27, no. 10, pp. 25-33, 2018.

[2] Y. Li, S. Yuan, and H. Lai, "Numerical study of unsteady flows with cavitation in a high-speed micro centrifugal pump," Journal of Thermal Science, vol. 26, no. 1, pp. 18-24, 2017.

[3] Y. Yuan and S. Yuan, "Analyzing the effects of splitter blade on the performance characteristics for a high-speed centrifugal pump," Advances in Mechanical Engineering, vol. 9, no. 12, Article ID 1687814017745251, 2017.

[4] H. Alemi, S. A. Nourbakhsh, M. Raisee, and A. F. Najafi, "Effects of volute curvature on performance of a low specific- 
speed centrifugal pump at design and off-design conditions," Journal of Turbomachinery-transactions of The Asme, vol. 137, no. 4, p. 041009, 2015.

[5] N. Casimir, X. Zhu, M. Hundshagen, G. Ludwig, and R. Skoda, "Numerical study of rotor-stator interaction of a centrifugal pump at Part Load with special emphasis on unsteady blade load," ASME Journal of Fluids Engineering, vol. 142, no. 8, Article ID 081203, 2020.

[6] B. Jafarzadeh, A. Hajari, M. M. Alishahi, and M. H. Akbari, "The flow simulation of a low-specific-speed high-speed centrifugal pump," Applied Mathematical Modelling, vol. 35, no. 1, pp. 242-249, 2011.

[7] B. Yang, B. Li, H. Chen, and Z. Liu, "Entropy production analysis for the clocking effect between inducer and impeller in a high-speed centrifugal pump," Proceedings of the Institution of Mechanical Engineers - Part C: Journal of Mechanical Engineering Science, vol. 233, no. 15, pp. 5302-5315, 2019.

[8] J.-M. Jin, K. J. Jung, Y.-H. Kim, and Y.-J. Kim, "Effects of gap between impeller and volute tongue on a pressure fluctuation in double-suction pump," Journal of Mechanical Science and Technology, vol. 34, no. 12, pp. 4897-4903, 2020.

[9] Y.-x. Xiao, Z.-w. Wang, J. Zhang, and Y.-y. Luo, "Numerical predictions of pressure pulses in a Francis pump turbine with misaligned guide vanes," Journal of Hydrodynamics, vol. 26, no. 2, pp. 250-256, 2014.

[10] C. Wang, Y. Zhang, Z. Li, A. Xu, C. Xu, and Z. Shi, "Pressure fluctuation-vortex interaction in an ultra-low specific-speed centrifugal pump," Journal of Low Frequency Noise, Vibration and Active Control, vol. 38, no. 2, pp. 527-543, 2019.

[11] S. Yuan, J. Zhang, Y. Tang, J. Yuan, and Y. Fu, "Research on the design method of the centrifugal pump with splitter blades," The Fluids Engineering Division Summer Meeting, vol. 1, p. 14, 2009.

[12] G. Song, L. P. Chua, and T. M. Lim, "Numerical study of a centrifugal blood pump with different impeller profiles," ASAIO Journal, vol. 56, no. 1, pp. 24-29, 2010.

[13] P. Fang, J. Du, and S. Yu, "Impeller (straight blade) design variations and their influence on the performance of a centrifugal blood pump," The International Journal of Artificial Organs, vol. 43, no. 12, pp. 782-795, 2020.

[14] B. Cui, D. Chen, C. Wang, Z. Zhu, Y. Jin, and Y. Jin, "Research on performance of centrifugal pump with different-type open impeller," Journal of Thermal Science, vol. 22, no. 6, pp. 586-591, 2013.

[15] X. Su, W. Jin, Z. Zu, Z. Li, and H. Jia, "Performance characteristics and energy loss analyses of a high-speed centrifugal pump with straight blades," Journal of Applied Fluid Mechanics, vol. 14, no. 5, pp. 1377-1388, 2021.

[16] L. Guo, J. T. Liu, L. Q. Wang, L. Jiao, and Z. F. Li, "Numerical analysis on pump turbine runaway points," IOP Conference Series: Earth and Environmental Science, vol. 15, no. 4, Article ID 042017, 2021. 